\title{
Low-energy electron scattering by deoxyribose and related molecules
}

\author{
Carl Winstead ${ }^{\text {a) }}$ and Vincent McKoy \\ A. A. Noyes Laboratory of Chemical Physics, California Institute of Technology, \\ Pasadena, California 91125
}

(Received 8 June 2006; accepted 10 July 2006; published online 17 August 2006)

\begin{abstract}
We apply first-principles computational methods to study elastic scattering of low-energy electrons by 2-deoxyribose and 2-deoxyribose monophosphate, which are of interest as components of the DNA backbone, and to tetrahydrofuran (THF), which has been studied as a deoxyribose analog. To investigate the dependence of the scattering process on the molecular conformation, we examine $C_{s}$ and $C_{2}$ conformers of THF as well as the planar $C_{2 v}$ geometry imposed in earlier calculations. There is little difference between the elastic cross sections determined at the nonplanar geometries, but there are noticeable differences between those results and the cross sections computed using the planar ring. By comparing results for tetrahydrofuran obtained with and without inclusion of polarization effects, we obtain energy shifts that are applied to the computed resonance positions for deoxyribose and deoxyribose monophosphate. (C) 2006 American Institute of Physics.
\end{abstract}

[DOI: $10.1063 / 1.2263824]$

\section{INTRODUCTION}

The observation that slow electrons induce single- and double-strand breaks in DNA (Refs. 1 and 2) has stimulated considerable research, both experimental and theoretical, on electron interactions with subunits of DNA and RNA, particularly the purine and pyrimidine bases. ${ }^{3}$ To date, constituents of the backbone have received relatively less attention. However, tetrahydrofuran (THF) has been studied recently ${ }^{4-9}$ as a model for low-energy electron interactions with 2-deoxyribose (DR), the furanose sugar that links phosphate groups in the DNA backbone. Experimental studies by Lepage et al. have looked at resonant vibrational excitation of gas-phase and thin-film THF by low-energy electrons, ${ }^{4}$ Zecca et al. have reported the total cross section (TCS) for electron (and positron) scattering ${ }^{5}$ by gas-phase THF, and the differential cross section (DCS) for elastic scattering at $20 \mathrm{eV}$ and above was recently measured by Milosavljević et $a{ }^{6}{ }^{6}$ Two recent theoretical studies ${ }^{8,9}$ apply high-level methods to low-energy electron scattering by THF. Bouchiha et al. used the $R$-matrix method to study elastic and electronically inelastic scattering up to $10 \mathrm{eV},{ }^{8}$ while Trevisan et al. applied the Kohn method to elastic scattering up to $15 \mathrm{eV} .{ }^{9}$ In each of these theoretical studies, the molecular geometry is approximated by forcing the furanose ring to be planar so that $C_{2 v}$ symmetry may be used to simplify the calculations. The study by Możejko and Sanche ${ }^{7}$ is still more approximate, relying on both local potentials and the independent-atom model, and its results are therefore confined to intermediate and high energies. Trevisan et al. ${ }^{9}$ observed shape resonances in their elastic cross section, but Bouchiha et al. ${ }^{8}$ reported no shape resonances below $10 \mathrm{eV}$, and their integral cross section (ICS) does not agree well with the measured TCS. ${ }^{5}$

As far as we are aware, there have been no experimental

${ }^{a)}$ Electronic mail: carl@schwinger.caltech.edu or theoretical studies of low-energy electron scattering by DR itself. However, Ptasińska et al. ${ }^{10}$ studied dissociative attachment to and ionization of gas-phase deoxyribose by low-energy electrons. Antic et al. have also studied dissociative attachment ${ }^{11,12}$ and electron energy loss ${ }^{11}$ using thin films of deoxyribose analogs.

Because DR and THF pseudorotate ${ }^{13}$ among several configurations of nearly equal energy, an interesting issue is whether calculations at a single geometry are sufficient. If the cross section changes significantly as the molecular conformation changes, it may be necessary to average over molecular geometries to obtain reliable results. Moreover, it is plausible, but not certain, that THF is a good model for the larger and less symmetric DR, whose extra functional groups appended to the furanose ring might give rise to additional shape resonances. In this study, we address five main questions: Is THF a good model for DR? Is the planar $C_{2 v}$ geometry a good approximation for THF? More generally, how does the electron cross section of THF depend on conformation? Where do the shape resonances in THF and DR lie? How does the DR cross section change if we attach a phosphate group, as is found in DNA?

In the process of addressing these questions, we also seek to obtain accurate elastic cross sections for THF that may be compared to existing measurements of the TCS (Ref. 5) and the elastic DCS, ${ }^{6}$ as well as to obtain more approximate elastic cross sections for DR and deoxyribose monophosphate (DRMP) that may serve as a point of departure for future studies.

Our procedure is the following: First, working at a lower level of approximation, we compute elastic electronscattering cross sections for THF at two geometries that are local minima on the pseudorotation coordinate ${ }^{13}$ as well as at the planar-ring $C_{2 v}$ geometry, in order to investigate the dependence of the cross section on conformation. We then calculate cross sections at a higher level of approximation for an appropriate nonplanar-ring geometry of THF, in order to gain 

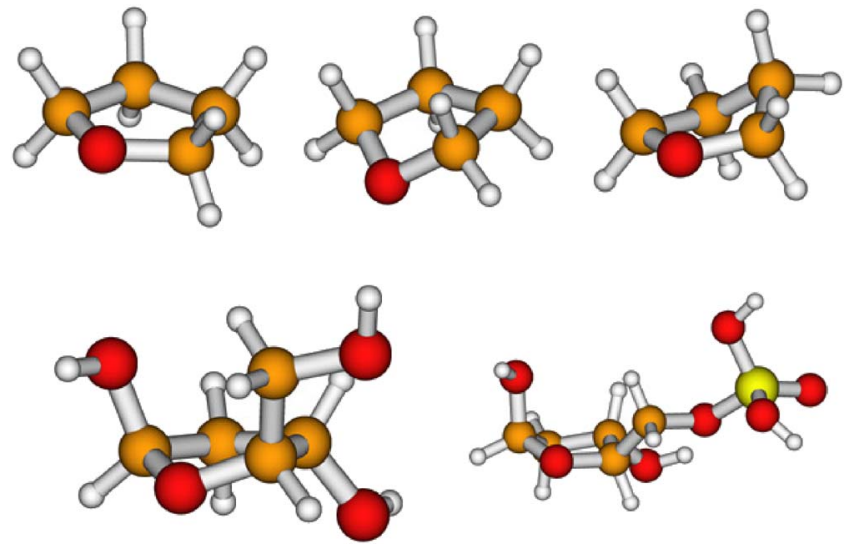

FIG. 1. (Color online) Structures employed for tetrahydrofuran, 2-deoxyribose, and 2-deoxyribose monophosphate in the present electronscattering calculations. Top row: for tetrahydrofuran, conformers belonging to the $C_{s}$ and $C_{2}$ point groups are shown center and right, along with the planar-ring geometry obtained from an optimization constrained to $C_{2 v}$ symmetry (left). Bottom row: deoxyribose (left) and its monophosphate derivative (right). Small white spheres represent hygdrogen, medium-shaded spheres carbon, and dark spheres oxygen; phosphorous is the light-shaded sphere surrounded by four oxygens at bottom right.

information on how the resonance positions shift. Lowerlevel calculations are then carried out for DR and DRMP, and the energy shifts determined from THF are applied to predict resonance positions for DR and DRMP.

Details of the calculations are given in the next section. Section III contains the results and discussion. Concluding remarks are given in Sec. IV.

\section{COMPUTATIONAL DETAILS}

The Schwinger multichannel $\operatorname{method}^{14,15}$ and its implementation $^{16,17}$ have been described elsewhere, so we give here only the particulars of the present calculations.

Geometries for THF, DR, and DRMP were optimized at the second-order Möller-Plesset level of theory using the electronic structure program GAMESS (Ref. 18) and its internal 6-31G $(d)$ basis set. The resulting geometries are shown in Fig. 1. For THF, we located minima of $C_{s}$ and $C_{2}$ symmetries (see Fig. 1) separated by 0.5 mhartree. At this level of theory, $C_{2}$ is lower in energy and so appears to be the global minimum. (More elaborate calculations ${ }^{13}$ place $C_{s}$ about 0.2 mhartree below $C_{2}$.) We also obtained a planar-ring geometry by performing a constrained optimization in $C_{2 v}$ symmetry. Although this $C_{2 v}$ geometry lies only 7.6 mhartree above the $C_{2}$ minimum, this small difference in total energy is the net effect of much larger changes in the electronic and nuclear energies - the nuclear repulsion energy, for example, changes by 1.7 hartree between $C_{2}$ and $C_{2 v}$ - and thus may understate the change in electronic structure.

Elastic electron scattering calculations were carried out for all of the species shown in Fig. 1 in the static-exchange (SE) approximation, wherein polarization effects are neglected. In these calculations, the target molecule's electron density was described in the restricted Hartree-Fock (RHF) approximation using the $\mathrm{TZV}++(3 d, 2 p)$ basis set as contained in GAMESS, ${ }^{18}$ with default splitting factors for the $d$ and $p$ polarization functions. The $3 s\left(x^{2}+y^{2}+z^{2}\right)$ linear com- bination of Cartesian $d$ orbitals was excluded, leaving a basis set of 33 contracted Gaussians per heavy atom and ten contracted Gaussians per hydrogen from which to form occupied orbitals describing the target and virtual orbitals describing the scattering electron. To capture a portion of the dipolescattering effect, we also carried out SE calculations for THF and deoxyribose using the $\mathrm{TZV}++(3 d, 2 p)$ basis set supplemented by four diffuse $s$ Gaussians (exponents $0.1,0.03$, 0.01 , and 0.003 ) and four diffuse $p$ Gaussians (exponents $0.3,0.1,0.03$, and 0.003 ) centered well outside the ring on the positive end of the molecule.

Calculations including polarization effects were carried out for the $C_{2}$ conformation of THF. In these static-exchange plus polarization (SEP) calculations, the $\mathrm{TZV}++(3 d, 2 p)$ basis set was again used, and the ground state of the target was again represented by the RHF wave function, but the variational space for the $(N+1)$-electron system was expanded to include configurations built on excited target configurations. In order to obtain a variational space of manageable size, we transformed the virtual orbitals into modified virtual orbitals $^{19}$ (MVOs) using a +6 cation Fock operator and only included virtual excitations from valence occupied orbitals into low-energy MVOs. ${ }^{20,21}$ Specifically, we allowed singletcoupled excitations from the ten lowest-energy valence orbitals into the ten lowest MVOs and coupled those $N$-electron singlets with the 40 lowest MVOs to form $(N+1)$-electron doublet configuration state functions (CSFs). From the five outermost valence orbitals, we allowed excitations into the 20 lowest MVOs and coupled the resulting singlets with any of the 240 MVOs to form doublet CSFs. This procedure resulted in variational spaces comprising $12669^{2} A$ and $13406{ }^{2} B$ CSFs, where $A$ and $B$ refer to irreducible representations of the $C_{2}$ point group.

In our implementation of the Schwinger multichannel (SMC) method, we represent free electrons with plane waves rather than with angular-momentum eigenfunctions (partial waves), both in evaluating the free-electron Green's function $^{14-17}$ and in evaluating the on-shell scattering amplitude. A partial-wave expansion of the scattering amplitude is, however, employed to obtain orientationally averaged differential and momentum-transfer cross sections. For small molecules and low impact energies, we have found order-23 Lebedev quadratures, ${ }^{22}$ with partial-wave expansions up to $\ell=14$ or 16 , adequate, but as the molecular size and/or the impact energy grows, larger quadratures and higher partial waves are required. The adequacy of both the quadrature and the partial-wave expansion is conveniently gauged from the fidelity with which the forward-scattering, backwardscattering, and integral cross sections obtained after partialwave expansion reproduce the corresponding quantities computed directly from the quadrature: if the partial-wave expansion is inadequate, agreement will be poor, and if agreement cannot be achieved for any size partial-wave expansion, the quadrature itself is inadequate. We have taken care in the present work to use converged on-shell quadratures. For THF and DR, we employed order-23 quadrature up to $20 \mathrm{eV}$ and order-35 quadrature, with partial-wave expansions up to $\ell=20$, above $20 \mathrm{eV}$; for DRMP, we employed order-35 quadrature at all energies below $50 \mathrm{eV}$ and order-47 


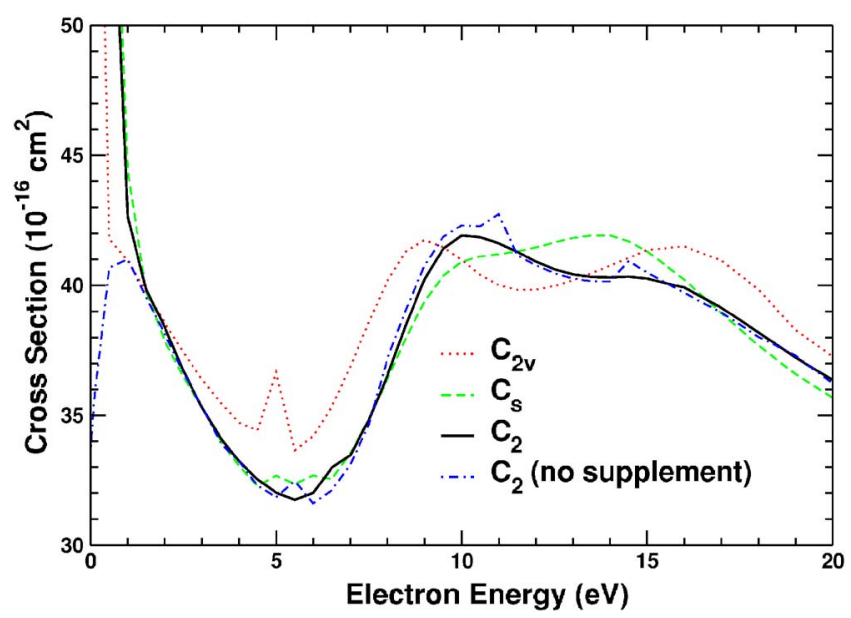

FIG. 2. (Color online) Integral elastic cross sections for electron scattering computed in the static-exchange approximation for different conformers of tetrahydrofuran. Dotted line: result for the planar-ring, $C_{2 v}$ geometry; dashed line: result for the $C_{s}$ conformer; solid line: result for the $C_{2}$ conformer; chained line: the $C_{2}$ result computed without extra diffuse functions at the positive end of the molecule.

quadrature, with expansions up to $\ell=25$, at $50 \mathrm{eV}$ and above. Much larger angular quadratures (up to order 89) were, of course, employed for evaluating the far-off-shell contributions to Green's function.

We did not include an explicit correction ${ }^{23}$ for longrange scattering by the permanent electric dipole moment in any of the present scattering calculations. At very low collision energies or extreme forward-scattering angles, therefore, we do not expect to obtain quantitative results. However, comparison between our calculations with and without a diffuse supplement, as well as between our results and those obtained by others with explicit dipole corrections, ${ }^{9}$ will allow us to make some judgments regarding the significance of dipole effects.

\section{RESULTS AND DISCUSSION}

\section{A. Tetrahydrofuran}

We begin by examining the dependence of the THF elastic cross section on conformation. Figure 2 shows the ICS for electron scattering by the $C_{2}, C_{s}$, and $C_{2 v}$ forms computed in the SE approximation. Small oscillations in the cross sections at $\sim 5,11$, and $14.5 \mathrm{eV}$ appear to be numerical noise due to basis-set limitations; the features of interest are the broad maxima associated with shape resonances. The $C_{2}$ and $C_{s}$ cross sections are very similar, though for $C_{s}$ the higherenergy peak is larger than the lower-energy peak, and vice versa for $C_{2}$. For $C_{2 v}$, we obtain two peaks of nearly equal height. However, these differences in magnitude are minor and only clearly visible because we have used a restricted ordinate in the figure. The second peak falls slightly lower in energy for $C_{s}$ than for $C_{2}$, but the main difference among the cross sections is that in the $C_{2 v}$ case the peaks are farther apart than in either $C_{2}$ or $C_{s}$ : the first peak shifts down in energy from $\sim 10$ to $\sim 9 \mathrm{eV}$, and the second shifts up from $\sim 14$ to $\sim 16 \mathrm{eV}$. Trevisan et al. ${ }^{9}$ also obtain a resonance

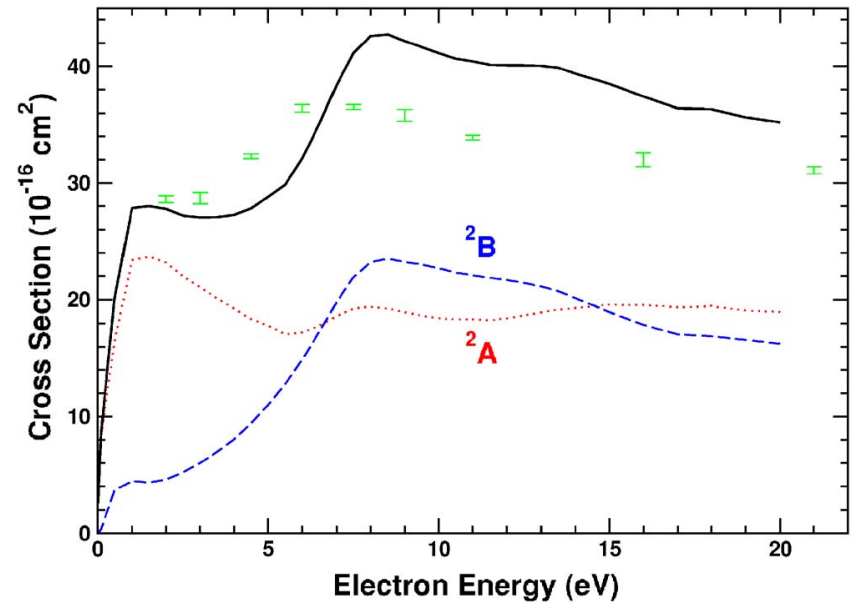

FIG. 3. (Color online) Integral cross section for elastic scattering of electrons by tetrahydrofuran with polarization effects included (solid line). Also shown are the contributions to the integral cross section from the ${ }^{2} A$ (dotted line) and ${ }^{2} B$ (dashed line) symmetries of the $C_{2}$ point group and the total scattering cross section measurements of Ref. 5 (data points with error bars).

peak (in the momentum-transfer cross section) near $9 \mathrm{eV}$ from SE calculations on the $C_{2 v}$ conformation; they do not report results at $16 \mathrm{eV}$.

For the $C_{2}$ conformation, Fig. 2 shows results both with and without supplementary diffuse functions at the positive end of the molecule. The only significant change in the cross section is at very low energy, where the results with the diffuse supplement increase in a manner typical of dipolar scattering, while the results without the supplement reach a maximum near $1 \mathrm{eV}$ and then decrease. Although not definitive, this comparison suggests that we are safe in neglecting dipole effects above $\sim 2 \mathrm{eV}$. Additional information on this point will be gained from the comparison of our SEP results to those of Trevisan et al., ${ }^{9}$ discussed immediately below.

SEP results for the elastic ICS obtained using the $C_{2}$ conformer of THF are shown in Fig. 3 together with their ${ }^{2} A$ and ${ }^{2} B$ components. Compared to Fig. 2, there is a clear downward shift in the resonance positions, as expected when the attractive polarization interaction is taken into account. A peak in the ICS now occurs at about $8.3 \mathrm{eV}$, while a shoulder lies between 13 and $14 \mathrm{eV}$. In each case, there is a downward shift of $\sim 2 \mathrm{eV}$ from the SE peak positions. Both the peak and the shoulder arise from overlapping ${ }^{2} A$ and ${ }^{2} B$ features.

The corresponding SEP momentum-transfer cross section is shown in Fig. 4 along with the results of Trevisan et al., ${ }^{9}$ which were calculated for a $C_{2 v}$ geometry. The agreement in magnitude is generally good except below $\sim 1.5 \mathrm{eV}$, where our result does not show the rise due to dipolar scattering. Our calculation places the maximum in the momentum-transfer cross section slightly lower in energy, reflecting a larger polarization shift in the present work $(\sim 2 \mathrm{eV}$ vs $\sim 0.5 \mathrm{eV})$, partially offset by the conformation effect on resonance positions seen in Fig. 2.

Electron energy-loss spectroscopy on gas-phase THF (Ref. 4) shows a broad peak at $8.4 \mathrm{eV}$, very close to the peak in our SEP ICS, in the excitation function for the $\nu_{2}$ vibrational mode. Because $\nu_{2}$ is a $\mathrm{C}-\mathrm{H}$ stretching mode, a shape 


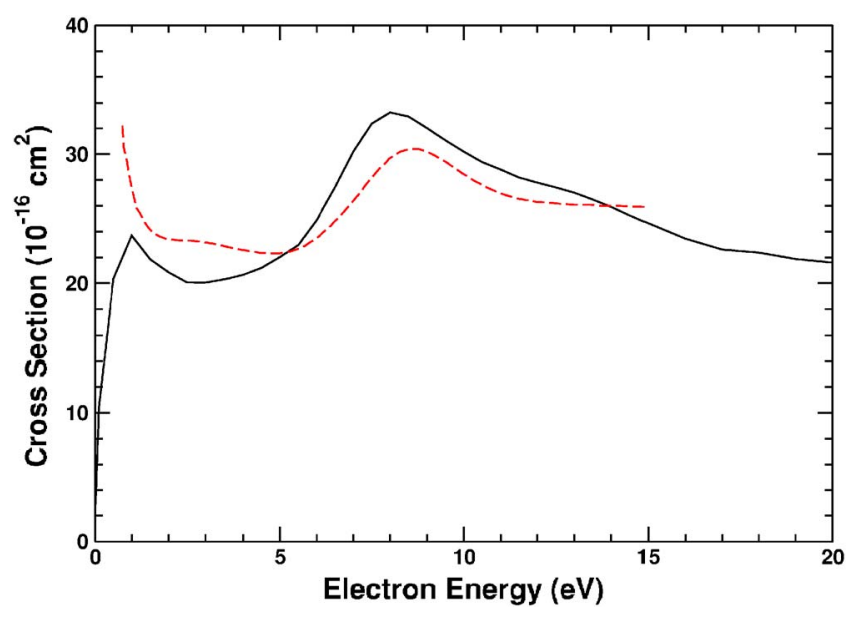

FIG. 4. (Color online) Momentum-transfer cross section for electron collisions with tetrahydrofuran. The solid line is the present result; the dashed line is from the $C_{2 v}$ calculation of Ref. 9 .

resonance at least partially of $\mathrm{C}-\mathrm{H} \sigma^{*}$ character seems to be implicated; however, the energy-loss spectrum at $8.5 \mathrm{eV}$ impact energy exhibits prominent features not only for $\nu_{2}$ but also for $\mathrm{C}-\mathrm{C}$ and $\mathrm{C}-\mathrm{O}$ stretching modes (as well as for several bends and torsions), complicating the resonance assignment. Based on our examination of minimal-basis-set orbital energies and corresponding wave functions, we believe that the peak at $8 \mathrm{eV}$ in the SEP elastic cross section is due to one or more shape resonances of primarily $\mathrm{C}-\mathrm{C}$ and $\mathrm{C}-\mathrm{O} \sigma^{*}$ character, while the shoulder between 13 and $14 \mathrm{eV}$ probably arises from overlapping resonances mostly of $\mathrm{C}-\mathrm{H} \sigma^{*}$ character, but definite assignments would require examination of the associated scattering wave functions (which our solution method does not generate) and/or the dependence of the features on nuclear geometry. We note that the clear presence of both features at the SE level rules out core-excited resonances as an explanation for them.

Figure 3 also shows the measured TCS reported by Zecca et al. ${ }^{5}$ Below the $8 \mathrm{eV}$ peak, our elastic ICS is in good agreement with the TCS. At higher energies, though, it appears that either our elastic ICS is somewhat high or the reported TCS is somewhat low, especially considering that the TCS at those energies may include significant contributions from inelastic processes. It should be noted that the error bars shown on the measurements in Fig. 3 only include the statistical error and not the larger systematic errors, which Zecca et al. estimated to vary from $13 \%$ at lower energy to $3.5 \%$ at higher energy. ${ }^{5}$ The computed ICS reported by Bouchiha et al. ${ }^{8}$ (not shown) is much larger than our ICS and than the TCS at all energies but is fairly close to ours from 6 to $10 \mathrm{eV}$ if the large dipole correction applied by Bouchiha et al. to their results is omitted.

Differential cross sections (DCSs) for THF obtained from the SEP calculation are shown at selected energies in Fig. 5, along with experimental results ${ }^{6}$ and other theoretical results $^{7,9}$ where available. For comparison we also show some of the results that we obtained in the SE approximation. At 6, 8, and $10 \mathrm{eV}$, there is extremely good agreement between the results of Trevisan et al. ${ }^{9}$ and our SE results computed for the $C_{2 v}$ geometry, except in the forward direction where our results are affected by the absence of a dipole correction. At $20 \mathrm{eV}$, the results of Trevisan et al. agree in shape with but are larger than our $C_{2 v}$ SE results. At all energies, there are noticeable differences in angular pattern between the $C_{2 v}$ and the $C_{2}$ DCSs, though the differences are least at the resonant energy of $8 \mathrm{eV}$. The results of Trevisan et al. were obtained with a more restricted treatment of polarization than in the present work and generally resemble our $C_{2 v}$ SE results best, our $C_{2} \mathrm{SE}$ results somewhat less so, and our $C_{2}$ SEP results least, so it appears that both the differences in molecular geometry and the differing treatments of polarization account for the differences between the two calculations at 6 and $10 \mathrm{eV}$. At $20 \mathrm{eV}$, both calculations are fairly consistent with the measured DCS when the experimental uncertainty is taken into account. It should be noted that the error bars shown on the measured points in-

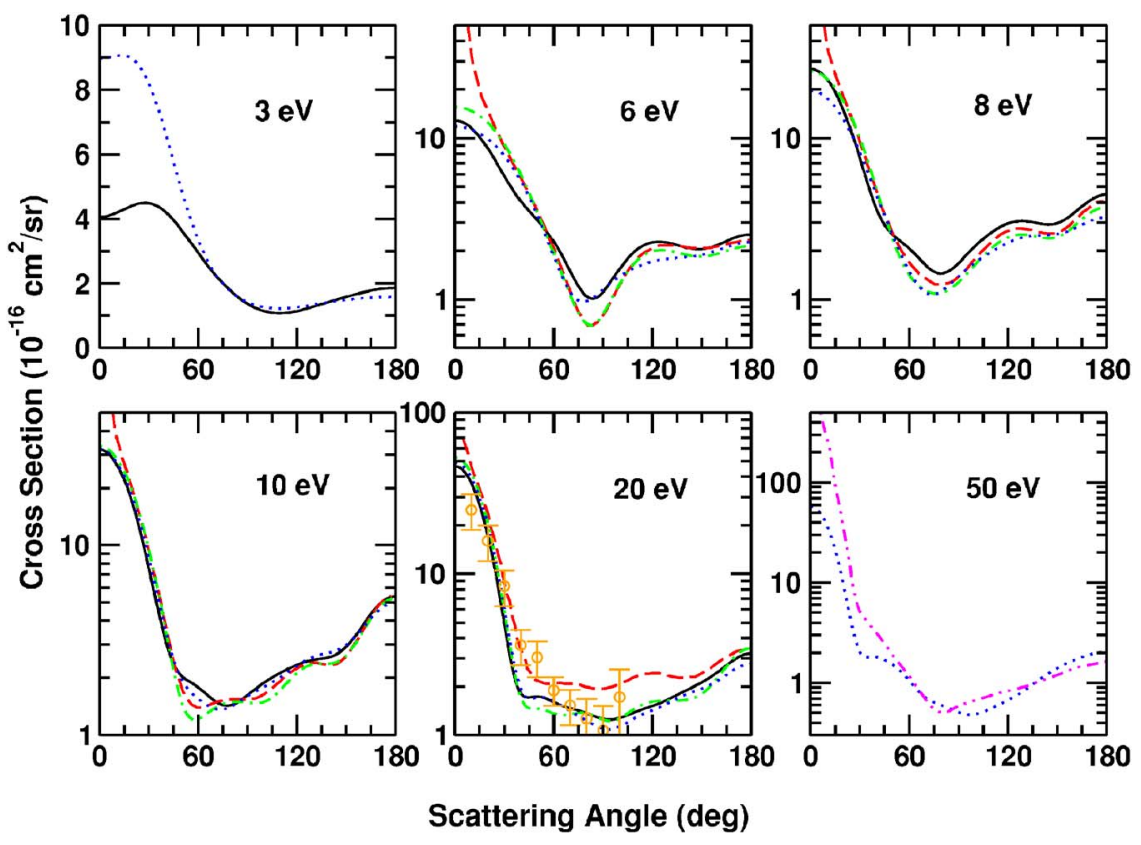

FIG. 5. (Color online) Differential cross sections for elastic electron scattering by tetrahydrofuran at various energies, as indicated. The solid line is the present result for the $C_{2}$ conformer including polarization and the dotted line the corresponding static-exchange result. The dotted-dashed line is the present static-exchange result for the $C_{2 v}$ geometry, while the dashed line is the Kohn calculation of Ref. 9. The double-dotted-dashed line at $50 \mathrm{eV}$ is the independent-atom result of Ref. 7; the circles at $20 \mathrm{eV}$ are measurements of Ref. 6. 


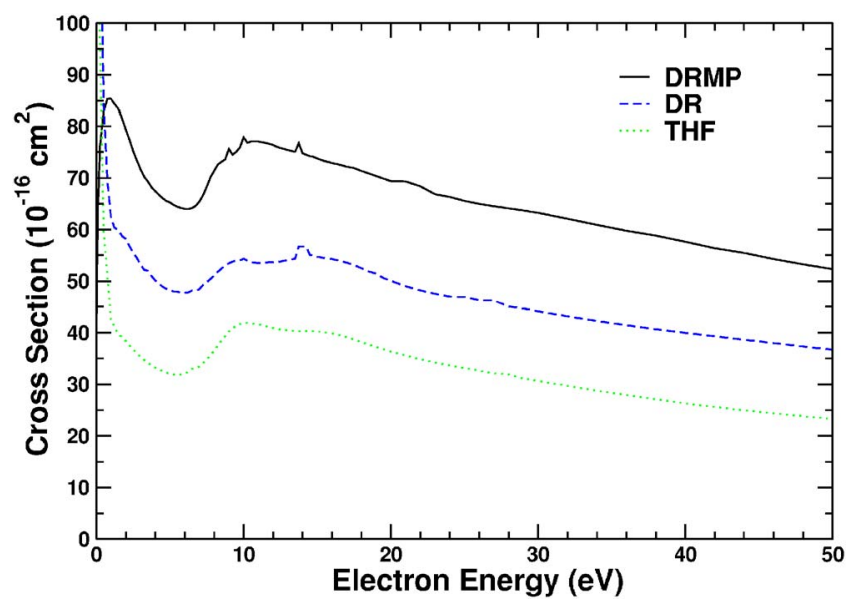

FIG. 6. (Color online) Integral elastic cross sections for electron scattering by 2-deoxyribose (dashes) and 2-deoxyribose monophosphate (solid line) computed in the static-exchange approximation. The static-exchange cross section for the $C_{2}$ conformer of tetrahydrofuran is also shown (dots) for comparison.

clude only statistical error and not the normalization uncertainty, which is estimated at $25 \% .^{6}$ At $50 \mathrm{eV}$, there is only rough agreement between our (SE) DCS and the independent-atom-model calculation. ${ }^{7}$

Despite these differences, the approximate agreement of our THF resonance position with experimental ${ }^{4}$ and other theoretical ${ }^{9}$ results provides some assurance that our SEP calculations yield good resonance positions for THF. Assuming (as we will see in the next subsection to be the case) that the elastic cross section for DR bears the expected close resemblance to that for THF, a downward shift of SE features by about $2 \mathrm{eV}$ to account for polarization effects will thus be appropriate for DR and DRMP. Moreover, the close similarity between the $C_{2}$ and $C_{s}$ cross sections for THF suggests that it is reasonable to base predictions for DR and DRMP on a single geometry.

\section{B. Deoxyribose and deoxyribose monophosphate}

The SE elastic ICS computed for DR and for DRMP are shown in Fig. 6. For comparison, we also show the SE cross section for THF. We are aware of no other calculated or experimental results for DR and DRMP. Setting aside an overall change in magnitude, there is a strong qualitative resemblance between the DR and THF results. For both molecules, the ICS exhibits a broad minimum near $6 \mathrm{eV}$ and broad maxima at about 10 and $16 \mathrm{eV}$. In DR the maxima appear, however, to be somewhat broader than in THF, likely reflecting the contribution of additional resonances, including $\mathrm{C}-\mathrm{O}$ and $\mathrm{O}-\mathrm{H} \sigma^{*}$ resonances arising from the hydroxyl groups. In DRMP, the resonances merge into a single broad maximum between 6 and $20 \mathrm{eV}$, with a somewhat sharper onset on the low-energy side than in DR or THF. The dropoff of the DRMP ICS at very low energy reflects the absence of supplementary diffuse functions (cf. the two $C_{2}$ calculations shown in Fig. 2). Sharp features in the DRMP and DR cross sections at $\sim 9-10$ and $14 \mathrm{eV}$ are numerical artifacts.

Momentum-transfer cross sections for DR and DRMP are shown in Fig. 7, again with THF SE results for compari-

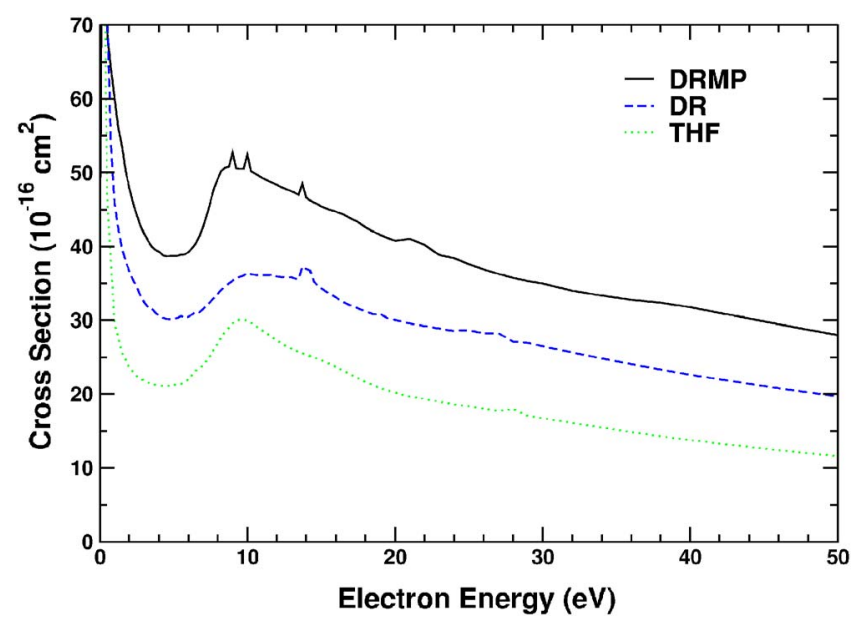

FIG. 7. (Color online) As in Fig. 6, for the momentum-transfer cross sections.

son. The momentum-tranfer results for each molecule very much follow the trend of the elastic ICS, with the exception that, in momentum transfer, the resonant enhancement near $8 \mathrm{eV}$ is relatively more prominent compared to the enhancement above $10 \mathrm{eV}$.

By applying the $2 \mathrm{eV}$ polarization shift determined for THF to the results in Fig. 6, we predict that the actual integral cross sections will show broad maxima at about 8 and $13 \mathrm{eV}$ for DR and a single, broader maximum at about $8 \mathrm{eV}$ for DRMP. For THF there was little change in the cross section magnitude between the SE and SEP results, so we anticipate little shift in the DR and DRMP peak heights. Similar remarks apply to the momentum-transfer cross sections of Fig. 7. The only experimental information we have on resonance positions in DR or DRMP comes from the dissociative-attachment measurements on DR by Ptasińska et $a l .{ }^{10}$ Besides several peaks at very low energies, they see a peak in the $\mathrm{C}_{3} \mathrm{H}_{5} \mathrm{O}_{3}^{-}$fragment-ion yield at $6 \mathrm{eV}$ and a set of three peaks in the $\mathrm{O}^{-}$yield at 7.3, 9.6, and $12.2 \mathrm{eV}$. Of these higher-energy features, those at 6 and $12.2 \mathrm{eV}$ are the strongest, with reported cross sections of $13.7 \times 10^{-18}$ and 3.1 $\times 10^{-18} \mathrm{~cm}^{2}$. Considering that attachment maxima usually appear to the low-energy side of elastic shape resonances owing to lifetime effects, ${ }^{24}$ the peak positions reported by Ptasińska et al. for DR are in reasonable agreement with our prediction of broad shape-resonant maxima at 8 and $13 \mathrm{eV}$, although it is also possible that the attachment features arise from core-excited resonances rather than from elastic shape resonances.

The elastic DCS for electron scattering by DR and DRMP is plotted at selected energies in Fig. 8. Because these results are obtained in the static-exchange approximation, we do not expect detailed agreement with the actual DCS at lower energies; however, comparison of the SE and SEP results for THF (solid and dotted curves in Fig. 5) suggests that the SE results for DR and DRMP should be reasonably close to SEP values above $\sim 5 \mathrm{eV}$. The DCS proves, as is typically true, more sensitive than the ICS to details of the scattering process: despite the general qualitative agreement between 


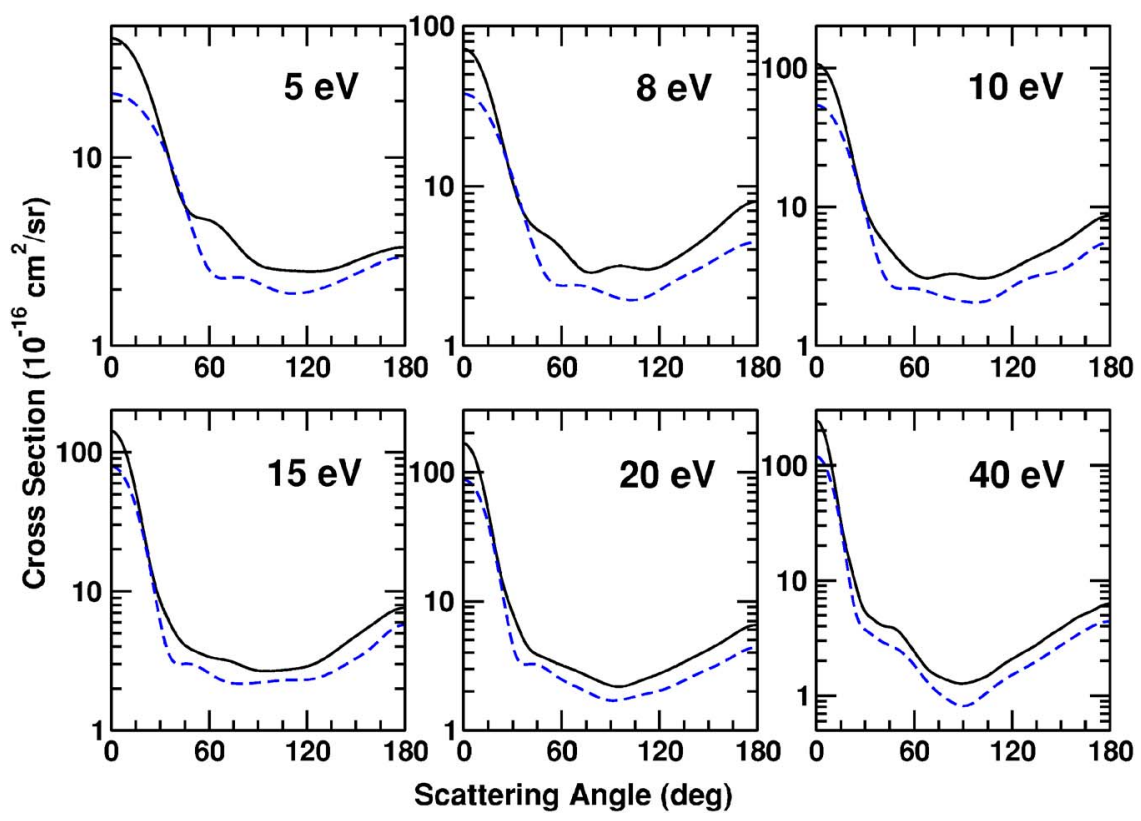

FIG. 8. (Color online) Differential cross sections for elastic scattering of electrons at the energies indicated, computed in the static-exchange approximation, for 2-deoxyribose (dashes) and 2-deoxyribose monophosphate (solid line).

the ICS for DR and that for THF, their DCS at a given energy tend to be quite different, and yet another angular pattern is observed for DRMP.

\section{SUMMARY AND CONCLUSIONS}

We have reported integral, differential, and momentumtransfer cross sections for elastic scattering by 2-deoxyribose and its monophosphate derivative, which occur in the backbone of DNA and whose cross sections are therefore relevant to studies of DNA damage by slow electrons. We have also examined the deoxyribose analog, tetrahydrofuran. Comparison between results obtained with and without polarization for THF establishes $2 \mathrm{eV}$ as an appropriate energy shift for correcting the peak positions in our SE integral cross sections for DR and DRMP. Comparison among electron cross sections obtained for THF at different nuclear geometries indicates that the $C_{2}$ and $C_{s}$ conformers give rise to very similar scattering behavior, while somewhat larger differences, including shifts in resonance positions, occur when the furanose ring is constrained to be planar. Differences between our results and earlier calculations ${ }^{7-9}$ can mostly be accounted for by consideration of the different approximations made. Experimental data for DR and DRMP would be very valuable, as would additional measurements for THF.

The general approach taken here of "calibrating" results for larger molecules obtained at the SE level by means of SEP calculations on smaller subunits should be applicable to other DNA moieties. Work is currently under way to apply the same approach to the nucleotide deoxyadenosine monophosphate. Experience gained on several such substructures should eventually help shape a more detailed view of lowenergy electron interactions with DNA itself.

\section{ACKNOWLEDGMENTS}

Sergio d'A. Sanchez performed preliminary calculations on deoxyribose while a visitor at Caltech. The authors gratefully acknowledge support of this work by the U.S. Depart- ment of Energy, Office of Basic Energy Sciences, and use of the computational resources of the Caltech-JPL Supercomputing Project.

${ }^{1}$ B. Boudaïffa, P. Cloutier, D. Hunting, M. A. Huels, and L. Sanche, Science 287, 1658 (2000).

${ }^{2}$ M. A. Huels, B. Boudaïffa, P. Cloutier, D. Hunting, and L. Sanche, J. Am. Chem. Soc. 125, 4467 (2003).

${ }^{3}$ For a recent review, see L. Sanche, Eur. Phys. J. D 35, 367 (2005).

${ }^{4}$ M. Lepage, S. Letarte, M. Michaud, F. Motte-Tollet, M.-J. HubinFranskin, D. Roy, and L. Sanche, J. Chem. Phys. 109, 5980 (1998).

${ }^{5}$ A. Zecca, C. Perazzolli, and M. J. Brunger, J. Phys. B 38, 2079 (2005).

${ }^{6}$ A. R. Milosavljević, A. Giuliani, D. Šević, M.-J. Hubin-Franskin, and B. P. Marinković, Eur. Phys. J. D 35, 411 (2005).

${ }^{7}$ P. Możejko and L. Sanche, Radiat. Phys. Chem. 73, 77 (2005).

${ }^{8}$ D. Bouchiha, J. D. Gorfinkiel, L. G. Caron, and L. Sanche, J. Phys. B 39, 975 (2006).

${ }^{9}$ C. S. Trevisan, A. E. Orel, and T. N. Rescigno, J. Phys. B 39, L255 (2006).

${ }^{10}$ S. Ptasińska, S. Denifl, P. Scheier, and T. D. Märk, J. Chem. Phys. 120, 8505 (2004).

${ }^{11}$ D. Antic, L. Parenteau, M. Lepage, and L. Sanche, J. Phys. Chem. B 103, 6611 (2000).

${ }^{12}$ D. Antic, L. Parenteau, and L. Sanche, J. Phys. Chem. B 104, 4711 (2000).

${ }^{13}$ A recent study of THF is V. M. Rayón and J. A. Sordo, J. Chem. Phys. 122, 204303 (2005).

${ }^{14}$ K. Takatsuka and V. McKoy, Phys. Rev. A 24, 2473 (1981).

${ }^{15}$ K. Takatsuka and V. McKoy, Phys. Rev. A 30, 1734 (1984).

${ }^{16}$ C. Winstead and V. McKoy, Adv. At., Mol., Opt. Phys. 36, 183 (1996).

${ }^{17}$ C. Winstead and V. McKoy, Comput. Phys. Commun. 128, 386 (2000).

${ }^{18}$ M. W. Schmidt, K. K. Baldridge, J. A. Boatz et al., J. Comput. Chem. 14, 1347 (1993).

${ }^{19}$ C. W. Bauschlicher, J. Chem. Phys. 72, 880 (1980).

${ }^{20}$ C. Winstead, V. McKoy, and M. H. F. Bettega, Phys. Rev. A 72, 042721 (2005).

${ }^{21}$ M. H. F. Bettega, C. Winstead, and V. McKoy, Phys. Rev. A 74, 022711 (2006).

${ }^{22}$ V. I. Lebedev and D. N. Laikov, Dokl. Akad. Nauk 366, 741 (1999); Dokl. Math. 59, 477 (1999), and references therein.

${ }^{23}$ T. N. Rescigno and B. I. Schneider, Phys. Rev. A 45, 2894 (1992), and references therein.

${ }^{24}$ L. G. Christophorou, D. L. McCorkle, and A. Christodoulides, in Electron-Molecule Interactions and Their Applications, edited by L. G. Christophorou (Academic, Orlando, 1984), Vol. 1, p. 477. Dissociation dynamics are discussed on pp. 504-505. 